\title{
Molecular ecology and phylogenetic systematics: Approaches to the conservation genetics of mammals
}

Mexico is considered as the fifth largest country in terms of global diversity; this attribute is linked to the heterogeneity of environments throughout the territory. The Mexican biota has many topics of interest to address, it only requires choosing a botanical or zoological group or any geographical region, but, above all, an academic question to answer. However, what does still remain to be known and understood? More than 5,000 species of mammals are known in the world, which possess biological, morphological, social, and reproductive adaptations that allow them to live in virtually all types of environments. At the global level, Mexico ranks third in terms of mammal diversity, with approximately 493 species described to date, in the understanding that the recognition, study and validity of species in natural environments are aspects not yet addressed.

With the aim to study and measure the diversity of mammal species, various topics and levels of ecological, geographical and genetic organization have been addressed. For this reason, a greater level of comprehensive and dynamic knowledge is required that allows the interconnection of the biodiversity components (ecological, biological and genetic elements). A core attribute of biodiversity is genetic diversity, in addition to evolutionary processes (mutations, migrations, gene flow, sexual reproduction, horizontal gene transfer, etc.). Therefore, the genetic component has been a re-valued tool used primarily in systematic biology. It is for this reason that disciplines such as molecular ecology and phylogenetic systematics have contributed to the generation of knowledge about the changes in the genetic structure of species and populations, with DNA- or RNA-based molecular genetic markers (proteins, sequences) as extremely useful tools.

The phylogenetic or cladistic systematics contributes to the interpretation of genealogical relationships between species and biological groups, supported on the similarities derived. The cladistic analysis is a biological classification system that groups together organisms according to their evolutionary relationships, being useful to resolve phylogenies. It is in the 1990s when there is a generation of biologists (including mammalogists) interested in using innovative tools (molecular markers, genetic and statistical algorithms) to understand ecological and evolutionary processes. Molecular techniques are evolving rapidly and have become supplementary tools in Biology, focusing on
1) phylogeny and phylogenetics; 2) evolutionary processes; 3) gene regulation and development analysis. 4) conservation genetics.

At the beginning of the 21st century there was an increase in studies focused on integrative taxonomy with the use of molecular tools, leading to the development of the information regarding the genetic library of the biota. The generation of bar codes (standardized DNA fragment to identify the species of all living organisms. In the case of animals, a fragment of the cytochrome oxidase I [COI], mitochondrial gene is used). It is worth remembering that the properties of mitochondrial DNA make it a marker with unique features, such as how to be inherited through the mother; a high mutation rate, which implies nucleotide substitutions; presents no genetic recombination; it is neutral; the distribution of haplotypes (sets of alleles that come from a single chromosome) is more influenced by demographic events in the history of the population through natural selection; it is amplified in a relatively simple way.

The emergence of multidiciplinary and international initiatives (www.barcodeoflife.org) focused on the generation of genetic information that will help to set the biota database at a global level. These initiatives have focused mainly on mega-diverse countries, so that Mexico could not be excluded, naming it as a BIOTRON node based on its biotic diversity and its potential to generate genetic libraries (www.boldsystems.org).

As a result of this project, there are currently a total of some 68,000 public records of mammal specimens with bar codes worldwide. It regards the information produced by 167 countries, highlighting the importance of 185 Scientific Collections, repositories of mammal specimens of study. The region that stand out for the entry of records is Latin America, although there is representation of countries from different continents (Table 1).

The groups of mammals with the largest number of records with genetic codes are primates $(22,356)$, with Russia as the country that has best documented this group. In second place, chiropterans, with 20,726 records, with the Cooperative Republic of Guyana as the country with the highest contribution to genetic records. The third re-presentative group is rodents, with 15,005 records, where Mexico has included the largest number of records (Table2), 
Table 1. Number of mammal records published in the website www.boldsystems.org (as of 24 January 2017). The number of orders, families and species by country is shown.

\begin{tabular}{lrrrr}
\hline Countries & Records & Orders & Families & Species \\
\hline Guyana & 7,229 & 8 & 26 & 171 \\
Mexico & 5,067 & 11 & 20 & 109 \\
Canada & 3,574 & 10 & 27 & 116 \\
Russia & 2,478 & 8 & 15 & 99 \\
Ecuador & 2,053 & 8 & 17 & 112 \\
Suriname & 1,933 & 4 & 13 & 108 \\
China & 1,896 & 10 & 25 & 172 \\
\hline
\end{tabular}

mostly belonging to the rodent families Heteromyidae and Cricetidae.

At the national level, some linking efforts have been directed to produce genetic information of mammal species; an example is the CONACyT "Red Temática" named "Barcode of Life in Mexico" (www.mexbol.org). Public information is currently available of more than 5,000 records of Mexican mammal species, including 11 orders (Rodentia, Chiroptera, Lagomorpha, Didelphimorphia, Carnivora, Soricomopha, Primates, Artiodactyla, Cingulata and Pilosa). The production of genetic sequences has been useful as a species conservation tool (NOM-059); as supplement and update of species inventories, as well as evolutionary work and recognition of species (taxonomic identification). An aspect that is worth noting regards the issues faced by the mammal species included in the Convention on International Trade in Endangered Species of Wild Fauna and Flora CITES Appendices, where the generation of genetic infor- mation allows resource managers to use a molecular tool to curb the wildlife trafficking (www.barcodeofwildlife.org species).

It is clear that we are witnessing the loss of environments at a global level, either by climate change or an anthropic effect, which is leading to the fragmentation or modification of natural habitats and, therefore, to changes in the distribution of wildlife species. In addition to the slow taxa

Table 2. Total number of records available for the most representative countries and orders in the genetic library. A) Cooperative Republic of Guyana; B) Mexico; C) Canada; D) Russia; E) Ecuador; F) Suriname; G) China (www.boldsystems.org).

\begin{tabular}{lrrrrrrrr}
\hline \multicolumn{1}{c}{ Order } & Total & A & B & C & D & E & F & G \\
\hline Primates & 22,356 & 4 & 16 & 66 & 652 & 12 & -- & 137 \\
Chiroptera & 20,726 & 6,138 & 1,000 & 899 & 354 & 1,575 & 1,616 & 572 \\
Rodentia & 15,005 & 945 & 3,563 & 1,165 & 1,048 & 423 & 283 & 717 \\
Carnivora & 2,373 & 15 & 90 & 137 & 49 & -- & -- & 5 \\
Soricomorpha & 2,261 & -- & 83 & 948 & 280 & -- & -- & 189 \\
Artiodactyla & 2,058 & 11 & 8 & 224 & 4 & -- & -- & 252 \\
Cetacea & 857 & -- & -- & 17 & -- & -- & -- & -- \\
Didelphimorphia & 633 & 113 & 93 & 8 & -- & 37 & 33 & -- \\
Lagomorpha & 525 & -- & 205 & 8 & 90 & 2 & -- & 15 \\
Perissodactyla & 522 & -- & -- & -- & -- & 1 & -- & 4 \\
\hline
\end{tabular}

identification process (we only know 10 to $12 \%$ of the total number of species). The decade of 2010 should be considered as the period of integral research in evolutionary biology as regards the study of the mammalian fauna, including comparative cytogenetic studies, ribosomal genes, microarrays and proteomics, gene mapping and genomes.

Patricia Cortés-Calva

Centro de Investigaciones Biológicas del Noroeste S. C. Av. Instituto Politécnico Nacional 195 La Paz 23096, Baja California Sur, México. 